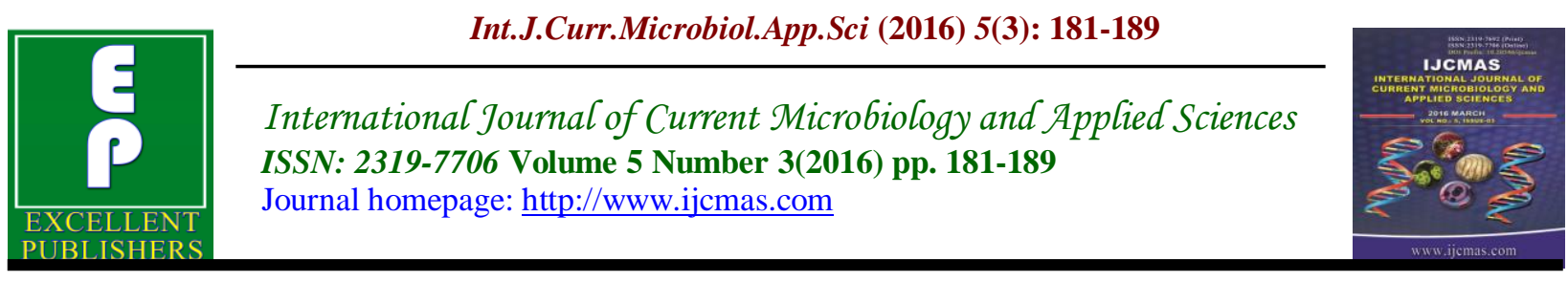

Original Research Article

http://dx.doi.org/10.20546/ijcmas.2016.503.022

\title{
Isolation and Identification of Protease Producing Bacteria from Food Processing Industries
}

\author{
I. S. Sony* and V. P. Potty \\ Department of Microbiology, Cashew Export promotion Council of India (CEPCI), \\ Mundakkal, Kollam, Kerala, India \\ *Corresponding author
}

\begin{tabular}{|c|c|}
\hline & A B S T R A C T \\
\hline Keywords & $\begin{array}{l}\text { The objective of the present study was to isolate and identify protease producing } \\
\text { bacteria from soil and wastewater collected from bakery industry especially halwa }\end{array}$ \\
\hline $\begin{array}{l}\text { Soil, } \\
\text { Waste water, } \\
\text { Bacteria, } \\
\text { Protease, } \\
\text { Gelatine agar, } \\
\text { Biomerieux, } \\
\text { VITEK } 2 \text { system. }\end{array}$ & $\begin{array}{l}\text { manufacturing units in Kollam \& Karunagappally region (Kerala), India and } \\
\text { detection of protease production on gelatine agar plates. Samples were serially } \\
\text { diluted and } 0.1 \mathrm{ml} \text { was spread on nutrient agar plates at } 37^{\circ} \mathrm{C} \text { for } 48 \text { hours. Total } 87 \\
\text { dissimilar colonies were selected and detected protease activity on gelatine agar } \\
\text { using } 0.8 \% \text { mercuric chloride and the activity was observed by the occurrence of } \\
\text { clear zone around the isolates. Among the } 87 \text { isolates, } 27 \text { isolates were showed } \\
\text { zone formation around colonies and } 8 \text { isolates were selected based on zone }\end{array}$ \\
\hline Article Info & $\begin{array}{l}\text { TKMFT39, TKMFT53, TKMFT61.The selected were identified using cultural } \\
\text { characterization, microscopic observation and biochemical identification using }\end{array}$ \\
\hline $\begin{array}{l}\text { Accepted: } \\
\text { 15 February } 2016 \\
\text { Available Online: } \\
\text { 10, March } 2016\end{array}$ & $\begin{array}{l}\text { Biomerieux VITEK } 2 \text { system as Cedecea davisae, Staphylococcus intermedius, } \\
\text { Enterobacter asburiae, Alloiococcus otitis and Proteus mirabilis. These bacterial } \\
\text { isolates can be use as microbiological tool for recycling of waste from food } \\
\text { processing industries. }\end{array}$ \\
\hline
\end{tabular}

\section{Introduction}

Proteases are a group of enzymes catalyze hydrolysis of polypeptide chains into smaller polypeptides or free amino acids. Huge percentages (Ca.59\%) of the international market of industrially important enzymes are occupied by proteases (Deng et al., 2010) Proteases represent the important group among the industrial enzymes with a long array of industrial applications. (Ashis and Sudhir, 2011; Singhal et al., 2012; Silva et al., 2012; Roja Rani et al., 2012;
Sankeerthana et al., 2013; Adriana et al., 2013; Global Industrial Enzyme Market Research: 2013). Microbial proteases are one of the important groups of industrially and commercially produced enzymes and the initial screening methods for detection of protease production are of greatest importance (Kasana et al. 2011). Protease production is an inherent capacity of all microorganisms. (Padma Singh et al., 2015)Microbial sources play a major role in the production of all the three important 
types of proteases - acidic, neutral and alkaline. Proteases have tremendous applications, especially in the detergents, food processing industries, waste treatment in the waste management system, leather tanning, and Chemical industries as well as in metal recovery. (V.N.Jisha et.al.2013)

Bakery industry is the rapidly growing industry in our country and it is considered to be one of the sources of food industry wastes. In many food processing units, separation of useful food constituents from undesired one generates huge fraction of solid waste in the initial stage of processing (Abdalla S.M. Ammar,2014). Halwa is a sweet Indian dish made up of different substrates primarily maida-the finely milled and refined wheat flour. Major Halwa manufacturing units in Kerala are located in Kollam and Karunagappally region. The waste from halwa manufacturing units comprises of gluten which is obtained from maida flour in the initial stage of processing. The two viable alternatives for waste management are application of waste as animal feed and to landfill. (Brown et al.1989).

In the present study, soil was collected from gluten waste discharged soil of halwa manufacturing units in Kollam and Karunagappally region as well as waste water from the processing centres for isolation and identification of protease producing bacteria. Protease producing bacteria can be used for the degradation of gluten waste.

\section{Materials and Methods}

\section{Sources of Sample Collection}

Soil (from the close premises of food processing centres) and water (waste water from the processing units) samples were collected and stored in sterile containers at $4 \mathrm{C}$ with date and time.

\section{Isolation of Protease Producing Bacteria}

The technique used for isolation of bacteria from soil and water samples was serial dilution agar plate technique described by Sjodahl et al. (2002).1 gram/ $1 \mathrm{ml}$ of soil and water sample was added to $9 \mathrm{ml}$ of sterile distilled water and performed serial dilution up to $10^{-6}$ dilution under aseptic environment of laminar airflow cabinet. From each dilution $0.1 \mathrm{ml}$ was spreaded on nutrient agar medium plates. Inoculated plates were incubated at $37^{0} \mathrm{C}$ for 48 hours. Nutrient agar slants of bacterial isolates were prepared and maintained at $4^{0} \mathrm{C}$.

\section{Screening for Best Strain Produced Protease}

87 dissimilar colonies (TKMFT01 to TKMFT 87) from nutrient agar plates were selected and were subjected to primary screening for the production of protease by plate assay using protease specific medium containing (g/l) glucose 1.0, K2HPO4 2.0, Peptone 5.0, gelatin15.0, and agar 15.After $24 \mathrm{~h}$ incubation at $28^{\circ} \mathrm{C}$, the clear zone diameters were measured by flooded the plates with mercuric chloride solution, this method was described as gelatine clear zone method (Abdel Galil, 1992).

\section{Identification of Protease Producing Bacteria}

\section{Cultural Characterization}

Bacterial isolates were subjected to microscopic observation to obtain the colony morphology according to size, pigmentation, form, margin, elevation and colour.

\section{Microscopic Observation}

The selected bacterial isolates were gram stained and observed under a light microscope in high power magnifying lens. 


\section{Qualitative Test for Protease}

Proteolytic activities of selected bacterial isolates were detected on the basis of formation of clear zones around the bacterial isolates. Gelatine agar plates were used for this purpose.

\section{Identification of Bacteria using Biomerieux VITEK 2 System}

The selected organisms were identified using Biomerieux VITEK 2 system and the test method was A O A C OMA 2012.02.and the selected protease producing bacterial isolates were identified as Cedecea davisae (TKMFT 8 ), Proteus mirabilis (TKMFT 19), Staphylococcus intermedius (TKMFT 22 TKMFT 10,TKMFT53 ), Enterobacter asburiae (TKMFT 39, TKMFT25) and Alloiococcus otitis (TKMFT 61)

\section{Results and Discussion}

\section{Screening and Isolation of Proteolytic Bacteria}

Microbial proteases are preferred to proteases from plant and animal sources, since they comprises of almost all desired characteristics for their applications in biotechnology field(Gouda et al.,2006). The proteolytic ability of 87 bacterial isolates from soil and waste water samples were evaluated using Gelatine agar medium as shown in Fig.2.

It was reported as appearance of clear zone (zone of hydrolysis) around bacterial colonies. Among 87 isolates 27 isolates were found positive based on zone of hydrolysis and out of them 8 isolates
(TKMFT 8, TKMFT10, TKMFT19, TKMFT22, TKMFT25, TKMFT39, TKMFT53, and TKMFT61) were chosen for further study based on diameter of zone of hydrolysis.

The results of bacterial isolates showing zone of inhibition (Diameter in $\mathrm{mm}$ ) are presented in Table.1 Arun Kumar Sharma (2015) reported that gelatine agar medium was best than skim milk agar medium for qualitative test of protease production because zone of hydrolysis were developed with more clarity in gelatine agar plates.

Alnahdi (2012) reported screening and isolation of 6 different bacterial strains on gelatine agar medium. Proteolytic activity was expressed as diameter of clear zone of hydrolysis around bacterial colonies. The results of zone of hydrolysis on Gelatine agar are presented in Table.1. Similar screening method using gelatine agar has been adopted earlier by Abirami et al.,2011;Geethanjali and Subash (2011); Sevine and Demirkan,(2011); Najla O Ayaz (2012), Smitha et al., 2012; Sinha et al, (2013).

\section{Identification of Protease Producing Bacterial Isolates}

The selected 8 isolates were identified using cultural characterization, microscopic observation and biochemical identification using Biomerieux VITEK 2 system. Among the identified species,TKMFT8 belonged to Cedecea davisae,TKMFT 10, TKMFT22, TKMFT53 to Staphylococcus intermedius, TKMFT 25, TKMFT 39 to Enterobacter asburiae, TKMFT61 to Alloiococcus otitis and TKMFT19 to Proteus mirabilis. The results are presented in Tables 2,3,4,5. 
Table.1 Bacterial Isolates Showing Zone of Inhibition (Diameter in Mm)

\begin{tabular}{|l|l|l|}
\hline SI No & Bacterial isolates & Diameter of Zone of hydrolysis(mm) \\
\hline 1 & TKMFT 8 & 7 \\
\hline 2 & TKMFT 19 & 8 \\
\hline 3 & TKMFT 22 & 15 \\
\hline 4 & TKMFT 39 & 11 \\
\hline 5 & TKMFT 61 & 10 \\
\hline 6 & TKMFT10 & 8 \\
\hline 7 & TKMFT25 & 9 \\
\hline 8 & TKMFT53 & 9 \\
\hline
\end{tabular}

Table.2 Results of Gram Staining and Colony Characters of Selected Isolates

\begin{tabular}{|c|c|c|c|c|c|c|c|c|}
\hline \multirow{2}{*}{$\begin{array}{l}\text { Sl } \\
\text { No }\end{array}$} & \multirow[t]{2}{*}{$\begin{array}{l}\text { Bacterial } \\
\text { isolates }\end{array}$} & \multirow{2}{*}{$\begin{array}{l}\text { Result of } \\
\text { Gram } \\
\text { staining }\end{array}$} & \multicolumn{6}{|c|}{ Colonv characters on Nutrient agar } \\
\hline & & & Size & Pigmentation & Form & Margin & Elevation & Texture \\
\hline 1 & TKMFT 8 & $\begin{array}{l}\text { Gram } \\
\text { negative }\end{array}$ & Small & No & Circular & Entire & Flat & Rough \\
\hline 2 & TKMFT 19 & $\begin{array}{l}\text { Gram } \\
\text { negative } \\
\text { cocci }\end{array}$ & Small & No & Circular & Entire & Undulate & Smooth, oily \\
\hline 3 & $\begin{array}{l}\text { TKMFT22, } \\
\text { TKMFT10, } \\
\text { TKMFT53, }\end{array}$ & $\begin{array}{l}\text { Gram } \\
\text { negative } \\
\text { cocci }\end{array}$ & Small & Yellow & Circular & Entire & Raised & Smooth \\
\hline 4 & $\begin{array}{l}\text { TKMFT } 39 \\
\text { TKMFT25 }\end{array}$ & $\begin{array}{l}\text { Gram } \\
\text { negative } \\
\text { cocci }\end{array}$ & $\begin{array}{l}\text { Moder } \\
\text { ate }\end{array}$ & No & Circular & Entire & Flat & $\begin{array}{l}\text { Rough, } \\
\text { cottony }\end{array}$ \\
\hline 5 & TKMFT 61 & $\begin{array}{l}\text { Gram } \\
\text { positive } \\
\text { cocci }\end{array}$ & Large & No & Irregular & Entire & Flat & Rough \\
\hline
\end{tabular}

Figure.1 Isolation of Organisms from Soil and Waste Water

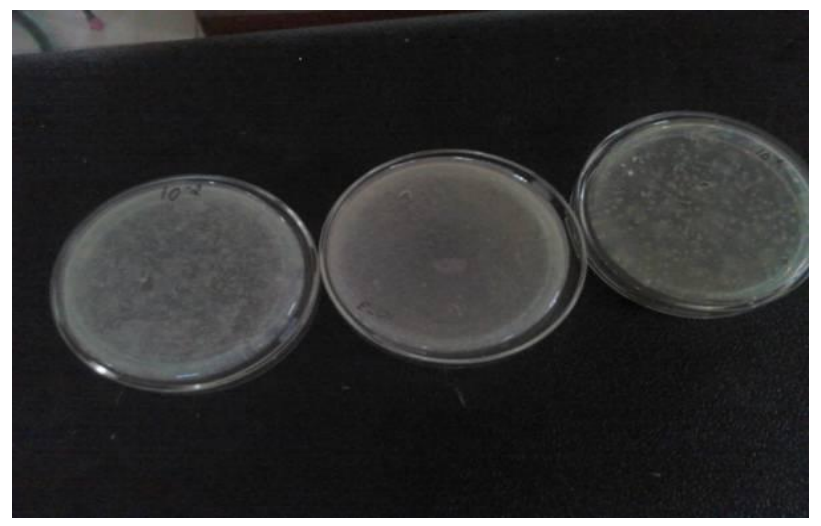


Table.3 Biochemical Details of Organisms Identified Using Biomerieux Vitek/Gn Cards

\begin{tabular}{|c|c|c|c|c|c|}
\hline \multirow[t]{2}{*}{ Well } & \multirow[t]{2}{*}{ Test } & \multirow{2}{*}{ Mnemonic } & \multicolumn{3}{|c|}{ Result } \\
\hline & & & TKMFT8 & TKMFT19 & TKMFT25,39, \\
\hline 2 & Ala-Phe-Pro-ARYLAMIDASE & APPA & - & - & - \\
\hline 3 & ADONITOL & ADO & - & - & - \\
\hline 4 & L-Pyrorolydonyl- & PyrA & - & - & - \\
\hline 5 & L-ARABITOL & IARL & - & - & - \\
\hline 7 & D-CELLOBIOSE & $\mathrm{dCEL}$ & - & - & + \\
\hline 9 & BETA-GALACTOSIDASE & BGAL & - & - & - \\
\hline 10 & H2S PRODUCTION & $\mathrm{H} 2 \mathrm{~S}$ & + & + & + \\
\hline 11 & BETA-N-ACETYL- & BNAG & + & + & + \\
\hline 12 & Glutamyl Arylamidase pNA & AGLTp & - & - & - \\
\hline 13 & D-GLUCOSE & dGLU & + & + & + \\
\hline 14 & GAMMA-GLUTAMYL- & GGT & + & + & + \\
\hline 15 & FERMENTATION/GLUCOSE & $\mathrm{OFF}$ & - & - & - \\
\hline 17 & BETA-GLUCOSID ASE & BGLU & + & + & + \\
\hline 18 & D-MALTOSE & dMAL & - & - & + \\
\hline 19 & D-MANNITOL & dMAN & + & - & + \\
\hline 20 & D-MANNOSE & $\mathrm{dMNE}$ & - & - & + \\
\hline 21 & BETA-XYLOSIDASE & BXYL & - & - & - \\
\hline 22 & BETA-Alanine arylamidase pNA & BAlap & - & - & - \\
\hline 23 & L-Proline ARYLAMIDASE & ProA & - & - & - \\
\hline 26 & LIPASE & LIP & - & - & - \\
\hline 27 & PALATINOSE & PLE & - & - & + \\
\hline 29 & Tyrosine ARYLAMIDASE & TyrA & + & + & + \\
\hline 31 & UREASE & URE & + & + & + \\
\hline 32 & D-SORBITOL & $\mathrm{dSOR}$ & - & - & + \\
\hline 33 & SACCHAROSE/SUCROSE & SAC & + & - & + \\
\hline 34 & D-TAGATOSE & dTAG & - & - & - \\
\hline 35 & D-TREHALOSE & dTRE & + & + & + \\
\hline 36 & CITRATE(SODIUM) & CIT & + & - & + \\
\hline 37 & MALONATE & MNT & - & - & - \\
\hline 39 & 5-KETO-D-GLUCONATE & $5 \mathrm{KG}$ & - & - & - \\
\hline 40 & L-LACTATE alkalinisation & ILATk & + & - & + \\
\hline 41 & ALPHA-GLUCOSIDASE & AGLU & - & - & - \\
\hline 42 & SUCCINATE alkalinisation & SUCT & + & - & + \\
\hline 43 & Beta-N-ACETYL- & NAGA & + & + & + \\
\hline 44 & ALPHA-GALACTOSIDASE & AGAL & - & - & - \\
\hline 45 & PHOSPHATASE & PHOS & + & + & + \\
\hline 46 & Glycine ARYLAMIDASE & GlyA & + & - & + \\
\hline 47 & ORNITHINE DECARBOXYLASE & ODC & + & + & + \\
\hline 48 & LYSINE DECARBOXYLASE & LDC & - & - & - \\
\hline 53 & L-HISTIDINE assimilation & IHISa & - & - & - \\
\hline 56 & COUMARATE & CMT & + & + & + \\
\hline 57 & BETA-GLUCORONIDASE & BGUR & & - & - \\
\hline 58 & O/129 RESISTANCE & O129R & + & + & + \\
\hline 59 & Glu-Gly-Arg-ARYLAMIDASE & GGAA & - & - & + \\
\hline 61 & L-MALATE assimilation & IMLTa & - & - & - \\
\hline 62 & ELLMAN & ELLM & - & - & - \\
\hline 64 & L-LACTATE assimilation & ILATa & - & - & - \\
\hline
\end{tabular}


Table.4 Biochemical Details of Organisms Identified Using Biomerieux Vitek/Gp Cards

\begin{tabular}{|l|l|l|l|l|}
\hline Well & Test & Mnemonic & \multicolumn{2}{|c|}{ Result } \\
\cline { 4 - 5 } & & & TKMFT22,10,53 & TKMFT61 \\
\hline 2 & D-AMYGDALIN & AMY & - & - \\
\hline 4 & PHOSPHATIDYLINOSITOL & PIPLC & - & - \\
\hline 5 & D-XYLOSE & dXYL & - & - \\
\hline 8 & ARGININE DIHYDROLASE 1 & ADH1 & + & - \\
\hline 9 & BETA-GALACTOSIDASE & BGAL & - & - \\
\hline 11 & ALPHA-GLUCOSIDASE & AGLU & - & - \\
\hline 13 & Ala-Phe-Pro ARYLAMIDASE & APPA & - & - \\
\hline 14 & CYCLODEXTRIN & CDEX & - & - \\
\hline 15 & L-Aspartate ARYLAMIDASE & AspA & - & - \\
\hline 16 & BETA GALACTOPYRANOSIDASE & BGAR & - & - \\
\hline 17 & ALPHA-MANNOSIDASE & AMAN & - & - \\
\hline 19 & PHOSPHATASE & PHOS & + & - \\
\hline 20 & Leucine ARYLAMIDASE & LeuA & + & - \\
\hline 23 & L-Proline ARYLAMIDASE & ProA & - & - \\
\hline 24 & BETA GLUCURONIDASE & BGURr & - & - \\
\hline 25 & ALPHA-GALACTOSIDASE & AGAL & - & - \\
\hline 26 & L-Pyrrolidonyl-ARYLAMIDASE & PyrA & - & - \\
\hline 27 & BETA-GLUCURONIDASE & BGUR & - & - \\
\hline 28 & Alanine ARYLAMIDASE & AlaA & + & - \\
\hline 29 & Tyrosine ARYLAMIDASE & TyrA & - & - \\
\hline 30 & D-SORBITOL & dSOR & - & - \\
\hline 31 & UREASE & URE & + & - \\
\hline 32 & POLYMIXIN B RESISTANCE & POLYB & + & - \\
\hline 37 & D-GALACTOSE & dGAL & + & - \\
\hline 38 & D-RIBOSE & dRIB & + & - \\
\hline 39 & L-LACTATE alkalinization & ILATk & + & - \\
\hline 42 & LACTOSE & LAC & - & - \\
\hline 44 & N-ACETYL-D-GLUCOSAMINE & NAG & + & - \\
\hline 45 & D-MALTOSE & dMAL & - & - \\
\hline 46 & BACITRACIN RESISTANCE & BACl & + & - \\
\hline 47 & NOVOBIOCIN RESISTANCE & NOVO & - & - \\
\hline 50 & GROWTH IN 6.5\% NaCl & NC6.5 & - & - \\
\hline 52 & D-MANNITOL & dMAN & - & - \\
\hline 53 & D-MANNOSE & dMNE & - & - \\
\hline 54 & METHYL-B-D-GLUCOPYRANOSIDE & MBdG & - & - \\
\hline 56 & PULLULAN & PUL & - & - \\
\hline 57 & D-FAFFINOSE & dRAF & - & - \\
\hline 58 & O/129 RESISTANCE (comp.vibrio.) & O129R & - & - \\
\hline 59 & SALICIN & SAL & - & - \\
\hline 60 & SACCHAROSE/SUCROSE & SAC & - & - \\
\hline 62 & D-TREHALOSE & dTRE & + & - \\
\hline 63 & ARGININE DIHYDROLASE 2 & ADH2s & - & - \\
\hline 64 & OPTOCHIN RESISTANCE & OPTO & + & - \\
\hline & & & & - \\
\hline
\end{tabular}


Table.5 Results of Microbial Identification Using Biomerieux Vitek 2 System

\begin{tabular}{|l|l|l|l|}
\hline SI No. & Bacterial isolate & Organism & Test Method \\
\hline 1 & TKMFT22,10,53 & Staphylococcus intermedius & VITEK/GP CARDS \\
\hline 2 & TKMFT25,39 & Enterobacter asburiae & VITEK/GN CARDS \\
\hline 3 & TKMFT8 & Cedecea davisae & VITEK/GN CARDS \\
\hline 4 & TKMFT19 & Proteus mirabilis & VITEK/GN CARDS \\
\hline 5 & TKMFT61 & Alloiococcus otitis & VITEK/GP CARDS \\
\hline
\end{tabular}

Figure.2 Zone of Hydrolysis on Gelatine Agar
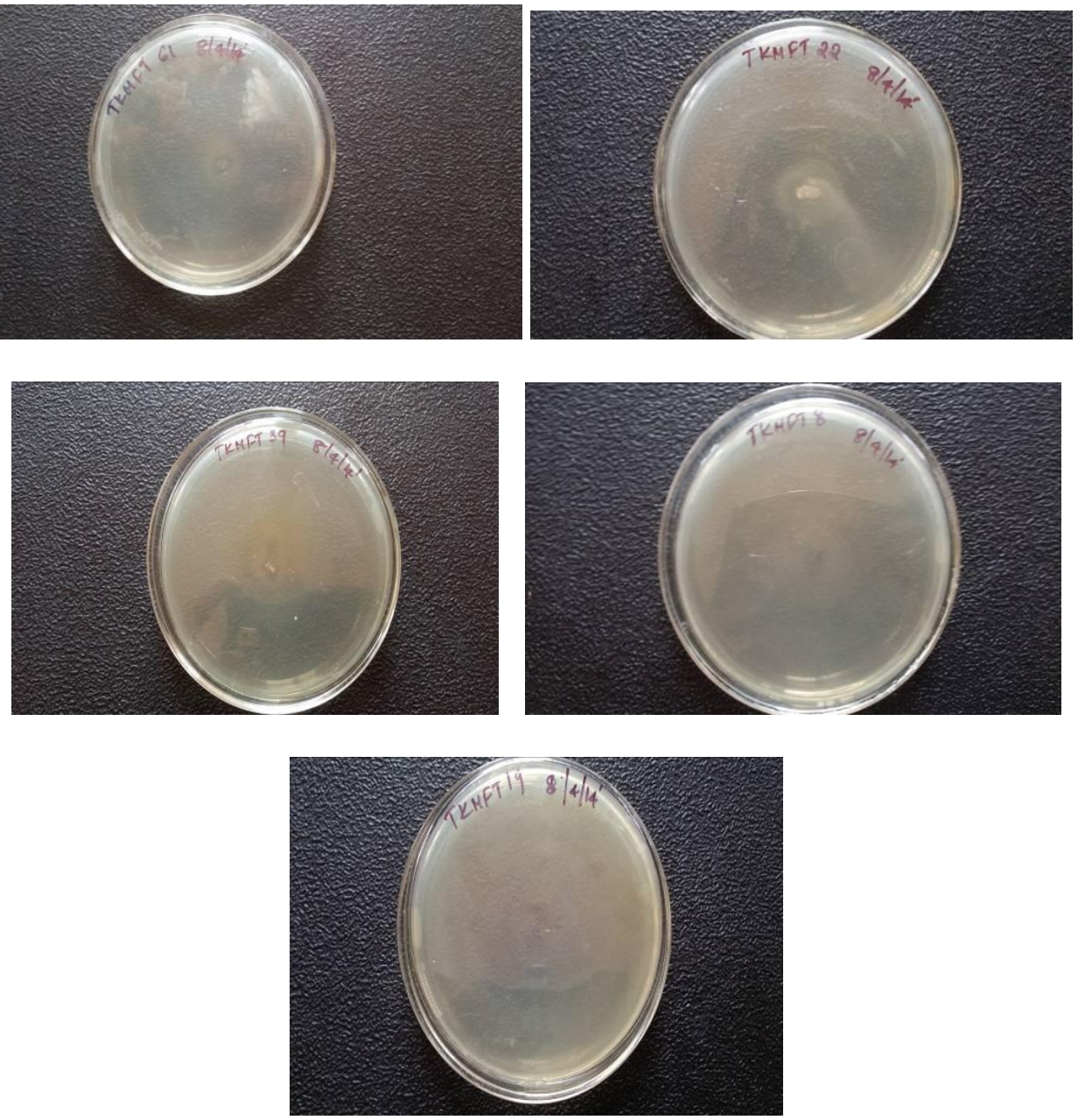

In conclusion, soil and waste water collected from food processing industries in Kollam and Karunagappally region shows presence of protease producers. A total number of 87 bacterial isolates were tested for protease production in gelatine agar plates and 27 isolates exhibited zone of hydrolysis and 8 isolates were selected based on zone 
diameter. All 8 isolates obtained by initial screening of protease production were identified based on cultural characteristics, microscopic observation and biochemical identification using Biomerieux VITEK 2 system, an automated microbiology system for identification of microorganisms. Among the 8 isolates, TKMFT22, 10, 53 are representing Staphylococcus intermedius, TKMFT25, 39 are representing Enterobacter asburiae, and TKMFT8, TKMFT 19, and TKMFT61 are representing Cedecea davisae, Proteus mirabilis and Alloiococcus otitis respectively according to the test results. Further these protease producing isolates can be used for degrading gluten waste from bakery industry viz.halwa manufacturing units. Hence the present study can play a significant role in the recycling of food industry wastes.

\section{Acknowledgement}

I would like to express my sincere thanks to The Head, Cashew Export Promotion Council of India (CEPCI), Mundakkal, Kollam, and Kerala, India for providing necessary research facilities.

\section{References}

Abdalla, S.M., Ammar. 2014. Food Processing Wastes: Characteristics, Treatments and Utilization Review. Qassim University. J. Agri. Vet. Sci., Vol.7(1): 71-84.

Abdel, Galil, O.A. 1992. Formation of proteases by Aspergillus fumigates and pencillium sp. J. King. Saud. Univ., Vol.4(2): 127-136.

Abirami, V., Meenakshi, S.A., Kanthymathy, K., Bharathidasan, R., Mahalingam, R., Panneerselvam, A. 2011. Partial Purification and Characterization of an Extracellular Protease from Penicillium janthinellum and Neurospora crassa. Eur. J. Exp. Biol., Vol.1(3): 114-123.
Adriana, C.F., Ruann, J.S.C., Maria, A.F., Antonio, S.E., Cristiane, S.F., Gustavo, A.S., Pinto. 2013. Canola cake as a potential substrate for proteolytic enzymes production by a selected strain of Aspergillus oryzae: selection of process conditions and product characterization. ISRN Microbiol., Vol.2013: 1-9.

Aihua, Deng, Jie Wu., Yun Zhang, Guoqiang, Zhang, Tingyi Wen. 2010. Purification and characterization of a surfactant-stable high alkaline protease from Bacillus sp. B001 Bioresource Technol., Vol.101(18): 7100-7106.

Alnahdi, H.S. 2012. Isolation and screening of extracellular proteases produced by new isolated Bacillus sp. J. Appl. Pharma. Sci., Vol.2(9): 071-074.

Arun Kumar Sharma, Vinay Sharma, Jyoti Saxena, Bindu Yadav, Afroz Alam, Anand Prakash. 2015. Isolation and Screening of Extracellular Protease Enzyme from Bacterial and Fungal Isolates of Soil. Int. J. Sci. Res. Environ. Sci., Vol.3(9): 0334-0340.

Ashis, K.M., Sudir K.R. 2011. A statistical approach for the enhanced production of alkaline protease showing the fibrinilytic activity from a newly isolated gramnegative Bacillus sp. strain AS-S20-I. New Biotechnol., Vol.28(2): 182-189.

Geethanjali, S., Subash, A. 2011. Optimization of Protease Production by Bacillus subtilis Isolated from Mid Gut of Fresh Water Fish Labeo rohita. World J. Fish and Marine Sci., Vol.3(1): 88-95.

Global Industrial Enzymes Market Report: 2013 Edition. http:/www.market researchreports.biz/analysis-details /global-industrialenzymes-market-report.

Gouda, M.K. 2006. Optimization and purification of alkaline proteases produced by marine Bacillus sp. MIG newly isolated from eastern harbor of Alexandria. Polish J. Microbiol., Vol.55(2): 119-126.

Kasana, R.C., Salwan, R., Yadav, S.K. 2011. Microbial proteases: detection, 
production, and genetic improvement. Crit. Rev. Microbiol., Vol.37(3): 262276.

Roja Rani, M., Prasad, N.N., Sambasivarao, K.R.S. 2012. Optimization of cultural conditions for the production of alkaline protease from a mutant Aspergillus flavus AS2. Asian J. Experimental Biol. Sci., Vol.3(3): 565-576.

Najla, O., Ayaz., 2012. Formation of proteases from newly isolated strain isolated from Saudi Arabia. J. Appl. Pharm. Sci., Vol.2(8): 190-193.

Singhal, P., Nigam, V.K., Vidyarthi, A.S. 2012. Studies on production, characterization and applications of microbial alkaline proteases. Int. J. Adv. Biotechnol. Res., Vol.3(3): 653-669.

Padma Singh, Anchal Rani, Neha Chaudhary. 2015. Isolation and characterization of protease producing Bacillus sp from soil. Int. J. Pharm. Sci. Res., Vol.6(4): 633639.

Pallavi Sinha, Rahul Kunwar Singh, Rishi Srivastva, Rajesh Sharma, Shree Prakash Tiwari. 2013. Characterization and optimization of alkaline protease enzyme produced by soil borne bacteria. Trends in Life Sci., Vol.2(2): 2319-4731.

Ronivaldo Rodrigues da Silva, Tatiana Pereira de Freitas Cabral, André Rodrigues, Hamilton Cabral. 2013. Production and partial characterization of serine and metallo peptidases secreted by Aspergillus fumigatus Fresenius in submerged and solid state fermentation. Brazilian J. Microbiol., Vol.44(1): 235243.

Sankeerthana Ca, Shabana Pinjara, Rizwana Tabassum Jambagia, Shamala Bhavimania, Anupama, S., Sarovar Ba, Shashikala, R., Inamdara. 2013. Production and Partial Characterization of Protease from Aspergillus flavus using Rice Mill Waste as a Substrate and its Comparison with Aspergillus Niger Protease. Int. J. Curr. Engi. Technol., 144-147.

Sevinc, N., Demirkan, E. 2011. Production of Protease by Bacillus sp. N-40 Isolated from Soil and Its Enzymatic Properties. $J$. Biol. Environ. Sci., Vol.5(14): 95-103.

Sjodahl, J., Emmer, A., Vincent, J., Roeraade, J. 2002. Characterization of proteinases from Antarctic krill (Euphausia superba). Protein Expression and Purification, Vol.26: 153-161.

Smita, G.S., Ray, P., Mohapatra, S. 2012. Quantification and Optimisation of Bacterial Isolates for Production of Alkaline Protease. Asian J. Exp. Biol. Sci., Vol.3(1): 180-186.

Veloorvalappil, N., Jisha, Robinson, B., Smitha, Selvanesan Pradeep, Sasidharan Sreedevi, Kizhak kepawothail, N., Unni, Sreedharan Sajith, Prakasan Priji, Moolakkariyil Sarath Josh, Sailas Benjamin. 2013. Versatility of microbial proteases, Vol.1(3): 39-51.

\section{How to cite this article:}

Sony, I.S., Potty, V.P. 2016. Isolation and Identification of Protease Producing Bacteria from Food Processing Industries. Int.J.Curr.Microbiol.App.Sci. 5(3): 181-189. doi: http://dx.doi.org/10.20546/ijcmas.2016.503.022 